\title{
Dinámica y
}

estructura de la

industria de las

tecnologías de la

información en

México

Pablo Mejía Reyes
El Colegio Mexiquense

\section{Consideraciones generales}

En términos de este documento definimos a la industria de las tecnologías de la información (ITI) como aquella encargada de producir y comercializar bienes y servicios destinados al almacenaje, procesamiento y transmisión de información, y comprende rubros tales como hardware, software y servicios. ${ }^{2}$ Es una de las más dinámicas de la economía mundial (Ernst y O’Connor, 1992), y sus usos han revolucionado la manera de hacer las cosas en casi todas las actividades humanas, con un especial impacto en la generación de bienes y servicios (Coriat, 1992). La permanente in-

\footnotetext{
${ }^{1}$ El autor agradece los comentarios de Zeus S. Hernández Veleros, Gerardo Moreno Ayala, Eduardo Loría Díaz y Henio Millán Valenzuela a versiones previas de este documento, así como los de dos dictaminadores anónimos de esta revista. También quisiera reconocer el apoyo brindado por la directora de esta revista, Alicia Lindón. La asistencia de investigación de Salomé López González y Alfredo Ríos Sara fue muy valiosa. Los errores y omisiones de este documento son responsabilidad del autor.

${ }^{2}$ No existe una definición ni una denominación universalmente aceptada de la industria que nos ocupa. Nos referiremos a ella como ITI cuando consideremos los tres rubros mencionados. En cualquier otro caso hablaremos específicamente de la producción de computadoras. De manera análoga, conforme a la denominación en las fuentes de información, nos referiremos a los bienes y servicios de la ITI también como bienes y servicios informáticos, y a las computadoras como equipo de cómputo o máquinas para el procesamiento de información. En todo caso, trataremos de ser específicos en el uso de cualquiera de estas denominaciones.
}

Economía, Sociedad y Territorio, vol. II, núm. 7, 2000, 497-532. 
novación tecnológica, traducida en la introducción de nuevos, poderosos y más baratos equipos, es uno de sus rasgos principales; y el crecimiento de sus ventas y de su comercio exterior la erigen como una de las empresas que se convertirán en soporte de la economía mundial.

La ITI es una de las industrias consideradas modernas, en la que la tecnología tiene un papel central. Precisamente el carácter de externalidad y bien público de la tecnología -lo cual favorece su transferencia y permite su estandarización- es lo que hace posible la entrada de nuevos participantes, al menos en segmentos que no exigen tecnología de punta. Sin embargo, la existencia de economías a escala -estáticas (tamaño mínimo eficiente para ingresar al mercado), dinámicas (de aprendizaje) y de alcance (plantas de multiproductos) - en las distintas etapas del proceso productivo, constituyen barreras a la entrada difíciles de salvar para los entrantes tardíos (Ernst y O'Connor, 1992), y vuelven factible la consolidación de monopolios u oligopolios (Tirole, 1988). La conjunción de ambos aspectos, la difusión de la tecnología y la existencia de economías a escala, han forzado a las empresas a emplear estrategias de competencia basadas en la diferenciación del producto y en la integración de paquetes de venta de equipo, programas, servicios y capacitación, dando lugar a un mercado que se aproxima más al de la competencia monopólica. El tránsito hacia un mercado de esta naturaleza ha sido paulatino. De un oligopolio firme y definido se ha pasado a otro altamente inestable, para llegar en algunos casos a uno muy cercano al de la competencia monopólica. Sin embargo, con el objeto de no perder la hegemonía, las empresas por tradición más fuertes han enfocado su atención hacia el control de la producción de insumos claves, el manejo de las redes de comercialización o la internalización de los productos de la investigación y desarrollo (IyD).

Aunque inminente, la difusión de la tecnología no es instantánea ni homogénea. Las empresas que encabezan la industria pertenecen a países tradicionalmente avanzados o a otros en los cuales su impulso ha formado parte de políticas deliberadas de desarrollo (Flamm, 1987; Carliner, 1991). En el primer caso, autores como Krugman (1990b) y Krugman y Obstfeld (1988) han argumentado que cuando se trata de industrias productoras de bienes en las que hay economías a escala, los países se especializan en la producción del bien para el que tienen un mercado más grande. Krugman (1990a), por otra parte, considera que en la determinación del tipo de bien que producirá cada país inter- 
vienen factores históricos, culturales o accidentales (guerras, religión, descubrimientos científicos, etc.). Según esta lógica, en ausencia de fenómenos afortunados, a menos que se ponga en práctica una política sectorial deliberada y cuidadosa, los países que no tienen las condiciones mencionadas jamás podrán ingresar en esta industria.

Existen, sin embargo, excepciones de países moderadamente avanzados que producen y exportan equipos de cómputo, mostrando un comportamiento no consistente con lo antes planteado. La explicación de este hecho radica en el papel que han desempeñado las empresas transnacionales (ET). Como parte de su estrategia para ganar competitividad, las ET han integrado vertical u horizontalmente etapas de la producción realizadas en distintos países, con el fin de aprovechar las ventajas que ofrece el país huésped en las etapas específicas de la producción ubicadas ahí (Grunwald y Flamm, 1985:1-11), lo que les permite aprovechar las economías de escala obtenidas de la especialización en ciertas líneas de productos que emplean insumos similares o que solamente se diferencian por su aspecto externo.

Éstas son algunas de las características de la industria productora de computadoras en todo el mundo (Mejía, 1998a y 1998b). El objetivo de este trabajo es documentar estos aspectos para el caso de esta industria en México. Los hallazgos más importantes de este texto pueden resumirse en los siguientes términos: actualmente existe un mercado dinámico, aunque heterogéneamente distribuido, dominado por las ET dedicadas a la producción de bienes, cuya tecnología se ha estandarizado. Estos aspectos se examinan en las restantes secciones. En particular, en la segunda sección se analiza la dinámica de la demanda mediante el uso de información desagregada para hardware, software y servicios. En la tercera sección se emplea información correspondiente a las dos empresas líderes del mercado para analizar la concentración industrial y el grado de competencia de los segmentos de computadoras personales y servidores. En la cuarta sección se aborda la dinámica de la inversión y producción, así como del tipo de productos generados por las empresas radicadas en México. Por último, se establecen algunas consideraciones finales. 


\section{La dinámica de la demanda}

\subsection{Comportamiento general}

El comportamiento del mercado mexicano es muy similar al del internacional: muestra elevadas tasas de crecimiento -aunque decrecientes, indicando una mayor madurez-, fuerte tendencia hacia el uso de sistemas más pequeños y poderosos, importancia cada vez mayor de los servicios y del software ${ }^{3}$ y un corto ciclo de vida de los productos. Para la configuración de estas características, empero, fue necesario pasar por diferentes etapas.

El impulso del gobierno en la conformación de este mercado resultó de fundamental importancia. Además de haber introducido el uso de los equipos de cómputo hace casi tres décadas, desde 1977 el gobierno destinó cantidades crecientes de recursos a la demanda de bienes y servicios de informática, las cuales alcanzaron un máximo de 600 millones de dólares en 1991. Con todo, las necesidades financieras asociadas a la crisis económica de los años ochenta y la política de cambio estructural basada en la desregulación y reprivatización de las actividades económicas, determinaron el retiro sistemático del gobierno de las actividades productivas. Por consiguiente, la participación del sector público en el mercado de las TI se redujo a menos de 50\% a partir de 1984, manteniéndose casi en 40\% hasta 1991. Desde entonces se aprecia un cambio importante en la tendencia de la participación del gobierno en este mercado, debido a la profundización de la política de privatización de las empresas paraestatales y de la banca (INEGI, 1992:54): la participación del sector público en este mercado disminuyó aún más, para ubicarse en alrededor de 30\% en 1994. ${ }^{4}$

Por ende, al parecer es ahora el PIB el determinante central del comportamiento del mercado, sobre todo desde mediados de la década anterior. En general -medido en dólares de los Estados Unidos-, el mercado mexicano de las TI presenta tasas de creci-

El auge reciente que experimenta el software se debe entre otros factores al tipo de tecnología en la que se basa el funcionamiento del equipo. Por su menor costo, la arquitectura más usada es la RISC (reduced instructions set computer), la cual, como su nombre lo indica, realiza un conjunto reducido de instrucciones, pero la mejora en su rendimiento se logra con la aceleración en la ejecución de tales instrucciones. En consecuencia, el programa debe realizar funciones adicionales.

${ }^{4}$ Las fuentes de esta información son INEGI (1994:98-99) y Poder Ejecutivo Federal (1996:125), las cuales solamente reportan la gráfica sin la correspondiente información estadística. 
miento mayores en valor absoluto a las del PIB real. Este patrón se observa tanto para el equipo de cómputo durante el periodo 19811989 (cuadro 1) como para el mercado total de las TI entre 1989 y 1999 (gráfica 1, en la que se incluyen perspectivas a partir de 1995). ${ }^{5}$ De modo específico, no obstante, existen algunas fluctuaciones en el comportamiento de las tasas de crecimiento que pueden explicarse por fenómenos claramente identificados.

\section{Gráfica 1}

México: Mercado de tecnología de información, 1989-1999

(tasas de crecimiento)

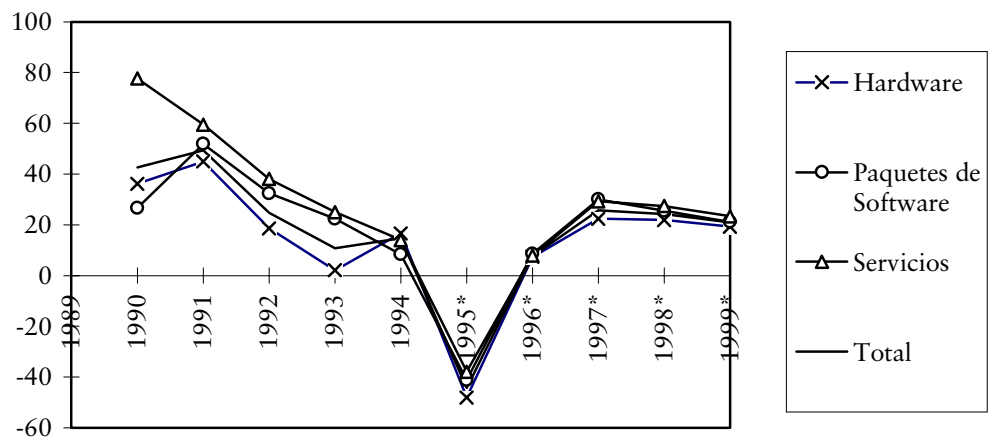

Fuente: Cálculos con información de Select-IDC.

Las drásticas caídas del mercado de cómputo en 1982-1983, 1986 y 1988, han estado asociadas con tasas de decrecimiento del PIB real, lo cual ha sido resultado de las crisis económicas experimentadas en esos años y/o de las políticas contraccionistas implantadas para paliar las crisis. La caída del mercado de las TI en 1994-1995, que se aprecia en la gráfica 1, se debe a razones similares. Esta conducta muestra la estrecha relación entre el PIB y el comportamiento del mercado de equipo de cómputo y de las TI.

Por otro lado, el retiro del gobierno, al parecer definitivo, de algunas actividades económicas, lleva a pensar que el peso de la administración pública federal en el mercado de computadoras se ha reducido de manera irreversible. Sin embargo, esta ins-

${ }^{5}$ Toda la información presentada en este capítulo, proporcionada por Select-IDC, está actualizada al 17 de enero de 1996. Esta división en dos subperiodos está determinada por la disponibilidad de la información. 


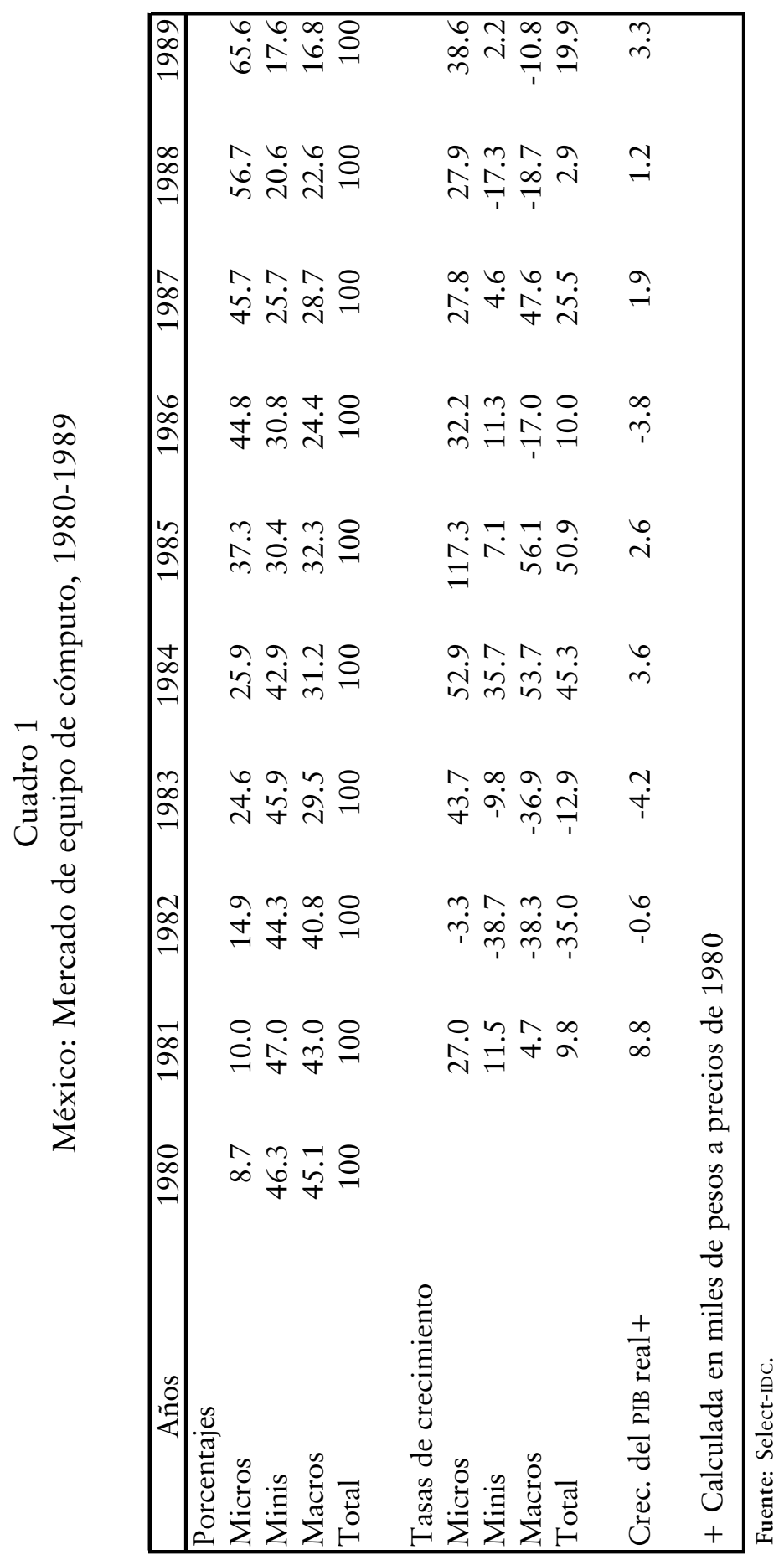


tancia como fuente de demanda no ha sido reemplazada por otros sectores y eso ha llevado a la desaceleración en el crecimiento del mercado a partir de 1991. A futuro sólo se espera una recuperación gradual encabezada por el sector privado e incentivada por el Programa de Desarrollo Informático (Poder Ejecutivo Federal, 1996).

Por otro lado, la distribución en el uso de bienes y servicios de las TI dentro de los distintos sectores institucionales muestra una gran heterogeneidad. Al parecer, dentro del sector público hubo un importante desaprovechamiento de los bienes y servicios de informática derivado de la gran desigualdad de su uso entre los distintos sectores de gobierno (Poder Ejecutivo Federal, 1996:30-32). Todavía en 1994 se notaban bastante tales diferencias: el sector central absorbía hasta 22\% y el paraestatal, $85 \%$, en tanto que la participación de los gobiernos estatales y municipales representaba sólo 16 por ciento.

Por otro lado, el sector privado actualmente explica la evolución de la mayor parte del mercado pues absorbe 70\% de la demanda doméstica. La necesidad de mejorar la eficiencia de sus actividades administrativas y comerciales, así como de automatizar sus procesos productivos, se ha incrementado debido a la liberalización del comercio y de la inversión extranjera directa. Estos cambios han exigido una mayor competitividad y han aumentado la cantidad de información sobre mercancías, proveedores, competidores, inversiones, etc., que debe manejarse.

Hasta ahora, la mayor parte de la demanda del sector privado corresponde a la de los grandes grupos comerciales, industriales y financieros; la de las empresas micro, pequeñas y medianas es casi nula (Poder Ejecutivo Federal, 1996: 43-46). Del análisis por sector (gráfica 2) se observa que el financiero es el que representa una mayor demanda debido a los grandes volúmenes de información que debe manejar y a la distancia, el tiempo y oportunidad exigidos por sus operaciones.

La demanda del sector industrial, por su parte, representa poco más de la quinta parte de la del sector privado. Principalmente, la introducción de la informática se da en dos ámbitos: la automatización de las operaciones de control y administración de las plantas, y la automatización de los procesos productivos. En particular, la industria automotriz es la que más utiliza la informática. Finalmente, el sector comercial está incorporando el intercambio electrónico de información por medio de redes para agilizar sus transacciones con clientes y proveedores. 
Gráfica 2

México: Uso de la informática por actividad económica, 1994

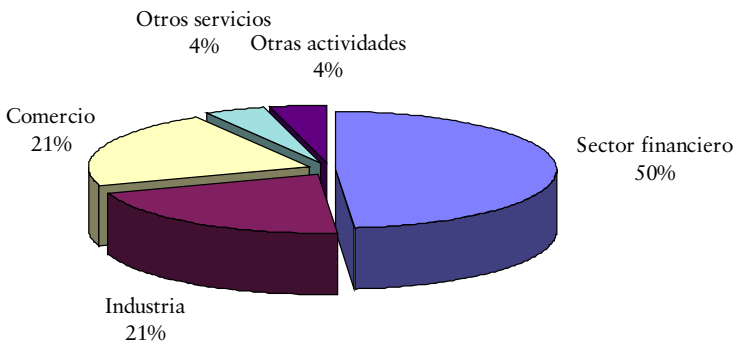

Fuente: Poder Ejecutivo

Aunque en el entorno global el gasto gubernamental y, principalmente, el PIB constituyen los determinantes centrales del mercado, en un ámbito de mayor desagregación pueden detectarse diferencias importantes en la dinámica de los mercados de los distintos productos.

\subsection{Comportamiento de los distintos segmentos del mercado}

Como se observa en el cuadro 1, durante el lapso 1981-1989, luego de la caída generalizada de 1982, el único rubro del hardware que ha presentado tasas de crecimiento positivas es el de computadoras personales (PC), cuyo máximo se alcanzó en 1985. Las ventas de minicomputadoras y de sistemas de gran escala han tenido un comportamiento irregular después de 1982, pero, salvo 1987, se aprecia un grave e irreversible deterioro. En los tres casos y en el total, sin embargo, la evolución de sus ventas muestra un cambio de tendencia a partir de 1985 cuando su crecimiento se reduce. Vale la pena destacar que en los episodios de decrecimiento o desaceleración, los sistemas de mayor tamaño han sido los más afectados.

A causa de las diferencias en la evolución de los distintos rubros, la estructura del mercado se ha invertido, lo cual puede apreciarse también en el cuadro 1. Después de que en 1980 un poco más de $90 \%$ se repartía igualmente entre minicomputadoras y sistemas de gran escala, en 1989 ambas sumaban casi 34\%. 
Por su parte, las PC pasaron de menos de 9 a más de $65 \%$ del total durante el mismo lapso.

En el segundo periodo, en el que se incluyen las perspectivas del mercado para el periodo 1995-1999, puede observarse un importante -aunque decreciente- aumento en las ventas hasta 1994, año seguido por la drástica caída de 1995. Se espera una lenta recuperación para 1996 y un crecimiento alto después, pero no a las tasas de 1990-1991 (véase gráfica 1). En este lapso, el análisis por tipo de producto sugiere un mayor dinamismo de los servicios, seguido de los programas para computadora y al final el hardware. Así, en el transcurso de esta década se ha dado una recomposición del mercado acorde con las tendencias mundiales. ${ }^{6}$ La primera columna del cuadro 2 muestra que hacia 1980 , $12.2 \%$ del mercado correspondía al software en paquete, $18.6 \%$ a servicios, y la diferencia (69.2\%) a hardware. La situación había cambiado en 1995 conforme a la última columna del mismo cuadro, en la que se muestra que los porcentajes correspondientes para ese año eran 12.9 , 34.1 y 53\%, respectivamente. Sin lugar a dudas, esta situación ha estado condicionada por las dificultades para desarrollar nuevas tecnologías ${ }^{7}$ y por la naturaleza misma de la arquitectura dominante (RISC), que exige el desempeño de más funciones por parte del software, así como por la aguda competencia que obliga a las empresas a promocionar sus productos en paquetes de hardware, software y servicios de soporte y capacitación (Ernst y O’Connor, 1992).

En el rubro de hardware, los sistemas personales ${ }^{8}$ son los de comportamiento más dinámico (gráfica 3), lo cual puede verse también en una menor participación dentro del total de los sistemas multiusuarios ${ }^{9}$ y el mantenimiento de la de sistemas de uso personal entre 1989 y 1995 (gráficas 4 y 5). Entre los sistemas multiusuarios existe cierto desplazamiento hacia los sistemas pequeños, aunque en general es menor la participación de

${ }^{6}$ La información presentada por Datamation para las empresas líderes de los Estados Unidos muestra que los cinco productos (de ocho) con mayor participación porcentual en el mercado mundial en 1994, fueron: servicios y mantenimiento, periféricos, PC, software y minicomputadoras; su participación respectiva fue de 27, 18, 16, 15 y 8 por ciento (Mejía, 1998b).

${ }^{7}$ Lo que ha llevado a sorprendentes alianzas entre empresas tradicionalmente competidoras para desarrollar nuevos productos, como el caso de IBM-Apple-Motorola que han desarrollado conjuntamente el microprocesador RISC Power PC (Salinas, 1993).

${ }^{8}$ Se refiere a los equipos usados por una sola persona, tales como PC y estaciones de trabajo, incluyendo además las adiciones a las PC.

9 Término usado para referirse a todos los sistemas de pequeña, mediana y gran escala, diferentes de las PC y las estaciones de trabajo. 
todos, resultante de la drástica caída de su tasa de crecimiento a partir de 1993 (véase también gráficas 3, 4 y 5).

Las ventas de PC son el rubro más estable de los sistemas de uso personal y del total del hardware. Aunque con una tendencia decreciente, es probable que después de la caída de 1995 ocurra una recuperación sostenida (gráfica 3). Sin embargo, es necesario hacer notar que la crisis de 1995 mostró que la demanda de PC ha dejado de ser inmune a las fluctuaciones económicas, y que su comportamiento se ha aparejado al de los demás rubros en este aspecto.

\section{Gráfica 3}

México: Rubros principales de hardware, 1989-1999

(tasas de crecimiento anual)
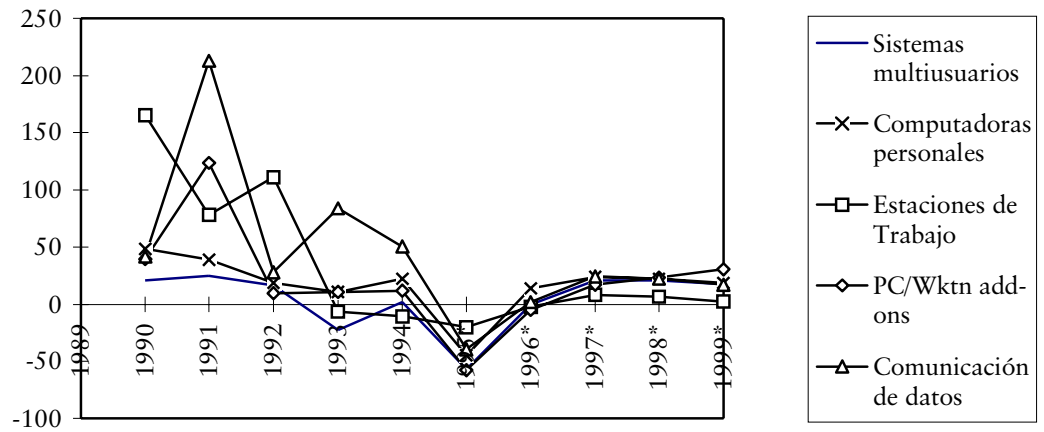

Fuente: Select-IDC.

Por su parte, las estaciones de trabajo tuvieron altas tasas de crecimiento a principios de los noventa y fueron uno de los sectores menos castigados por la crisis de 1994-1995, aunque no se espera un incremento sustancial de su participación en el total debido a los elevados costos asociados con la instalación de una red completa (gráfica 3). A su vez, las adiciones a las PC y/o estaciones de trabajo ${ }^{10}$ (PC/Wkts add-ons) constituyen un rubro muy sensible a las condiciones económicas generales, porque las adiciones a la infraestructura son de los primeros gastos pospuestos

${ }^{10}$ Gastos en hardware no adquiridos normalmente en una compra inicial y no incluidos en otras categorías, tales como: $a$ ) impresoras, $b$ ) actualización o sustitución de mecanismos de almacenamiento más cintas, y $c$ ) otras adiciones que incluyen actualización de la unidad central de procesamiento, sustitución o actualización de monitores y teclados, etcétera. 


\section{Gráfica 4 \\ México: Mercado de hardware, 1989 \\ (estructura porcentual)}

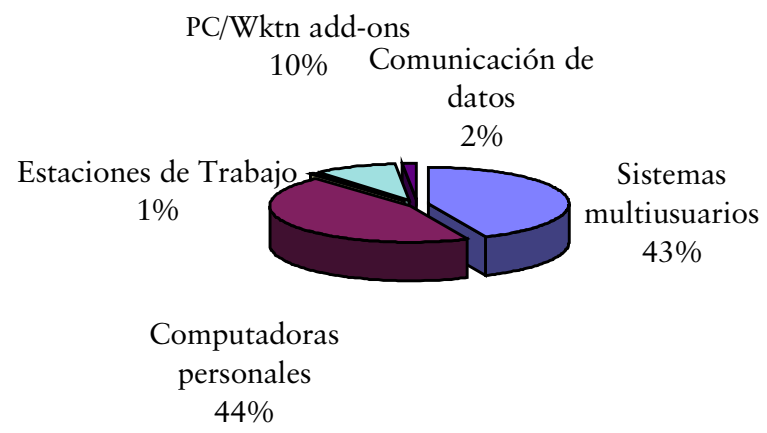

Fuente: Select-IDC.

\section{Gráfica 5}

México: Mercado de hardware, 1995

(Estructura porcentual)

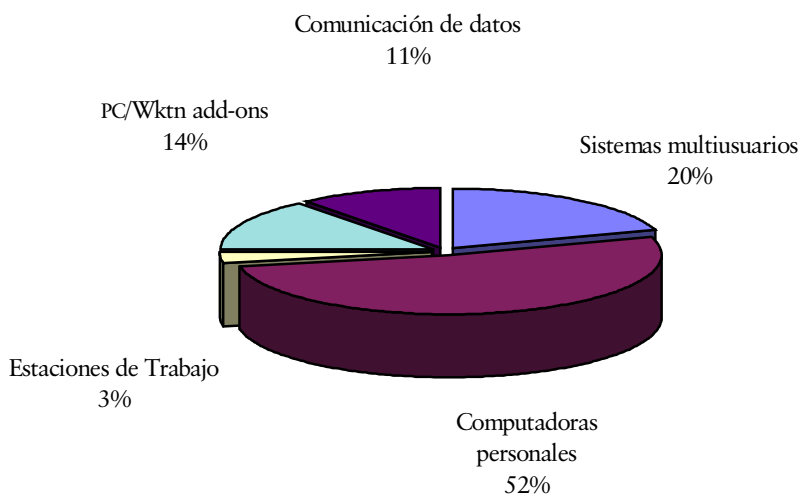

Fuente: Select-IDC. 
en épocas de crisis; su tasa de crecimiento es altamente volátil, aunque su participación en el total a lo largo del periodo es más o menos constante. El campo de comunicación de datos, finalmente, también muestra una evolución decreciente, con recuperación lenta luego de la crisis, aunque su participación porcentual podría ubicarse por debajo de $5 \%, 3.5$ puntos por encima de su participación inicial (véanse gráficas 3, 4 y 5).

Este desplazamiento hacia los sistemas pequeños se explica principalmente porque las características tecnológicas de sus procesos productivos y el enorme mercado potencial han obligado a las empresas a invertir en IyD para el mejoramiento en la capacidad, la confiabilidad y la versatilidad de tales equipos. Por otro lado, la difusión de la tecnología y las externalidades tecnológicas se han traducido en la aparición constante de nuevos competidores internacionales que han presionado los precios a la baja, pues en este segmento las barreras a la entrada se reducen de manera constante. Esta tendencia se ha acentuado-sobre todo en las corporaciones, instituciones de educación, oficinas públicas, etc.- con la generalización de las redes de computadoras que permiten compartir con varios usuarios los sistemas de alta potencia (Ernst y O’Connor, 1992).

El mercado de activos intangibles de la ITI se refiere al software en paquete ${ }^{11}$ y los servicios. Solamente se dispone de información para el periodo 1989-1999, pero es suficiente para matizar en el caso mexicano las tendencias en el mercado mundial y que ya se han explicado en un aspecto cualitativo (véase cuadro 2).

En el primer caso, el del software, se observa un comportamiento similar al del mercado nacional total, con una importante desaceleración a partir de 1990 y una recuperación moderada después de la crisis de 1995 (cuadro 2). Este comportamiento permitirá que para 1999 se mantenga la participación de ese rubro en el mercado en alrededor de $13 \%$, un poco más de $1 \%$ con respecto a la de 1989. Los servicios, por su parte, acusan también un comportamiento similar al del mercado total, aunque siempre con tasas de crecimiento mayores. Por ende, se espera que su participación se duplique en el total del mercado a lo largo del periodo considerado. Como se ha mencionado antes, estas ten-

${ }^{11}$ Se refiere a los programas comerciales disponibles para la venta o licencia por parte de vendedores de sistemas y vendedores independientes, y no incluye soluciones aplicativas de software agregadas a sistemas adquiridos de un manufacturero de hardware o de un tercero. 


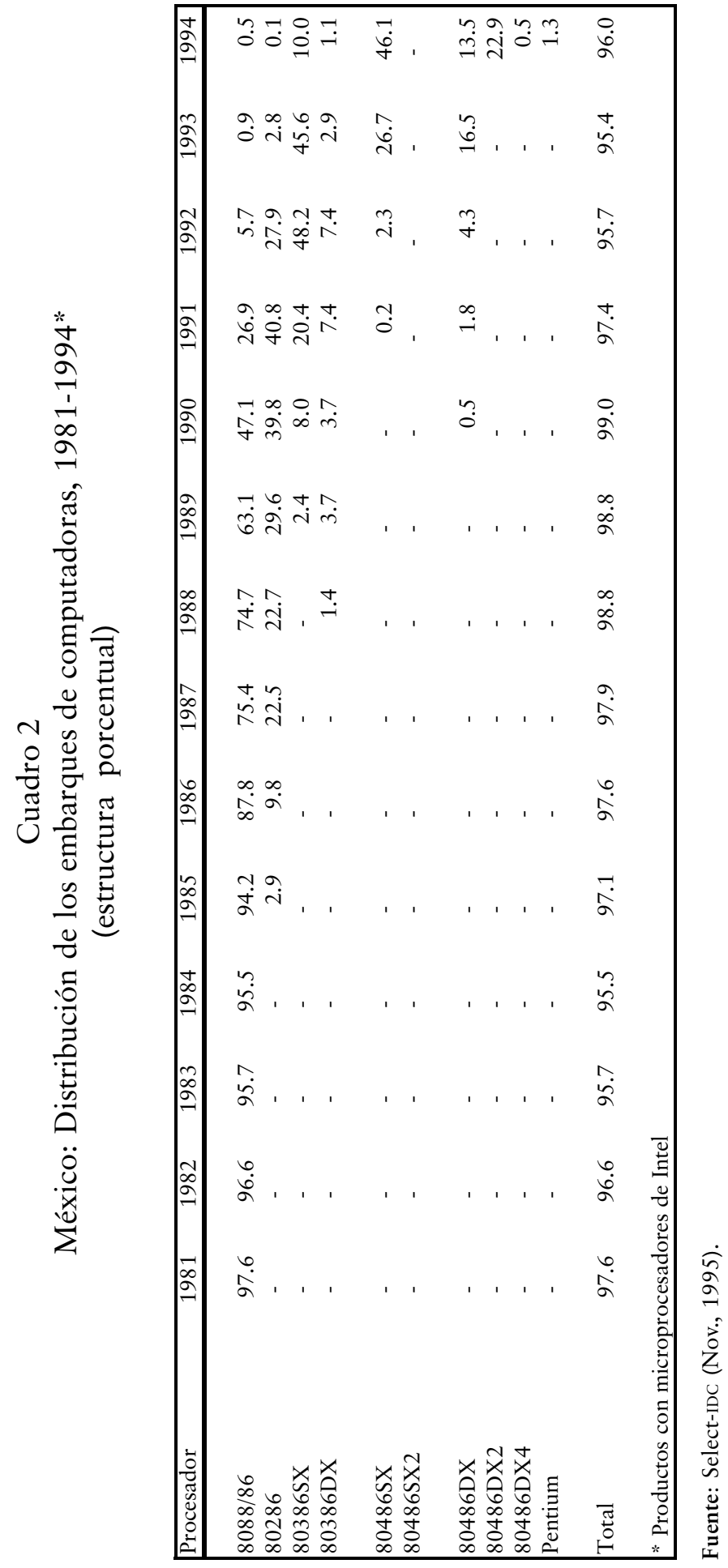


dencias se explican en buena medida por la creciente importancia que tienen ambos sectores en la integración de paquetes de venta que tienen como objetivo ofrecer un producto integral basado en un mayor acercamiento con el cliente para cubrir sus necesidades específicas.

El análisis por segmentos de mercado muestra que no habrá un cambio significativo en la estructura porcentual del software sino solamente una profundización de su perfil. Los sistemas y las utilerías ${ }^{12}$ seguirán siendo el factor menos importante, con una participación menor en $7 \%$ a la inicial. La participación del campo de herramientas ${ }^{13}$ se mantendrá aproximadamente igual, indicando la maduración de este tipo de programas. Finalmente, la participación de las soluciones aplicativas ${ }^{14}$ representará casi la mitad del software, lo que significa una mayor importancia de los programas a emplearse en actividades específicas.

El mercado de los servicios muestra que se observará en los próximos años una transformación radical en su estructura. Aunque sus tasas de crecimiento serán muy parecidas a las del mercado nacional, indicando que su importancia no será menor, la participación de los servicios de soporte ${ }^{15}$ se reducirá en 1999 a la mitad de la de 1989 (de 64\% a 29\%) debido al impresionante auge de los servicios profesionales, ${ }^{16}$ los cuales presentan tasas de crecimiento siempre superiores a las del total de los servicios y a

${ }^{12}$ Son programas diseñados principalmente para: 1) operar el equipo mediante sistemas operativos básicos y lenguajes de programación, incrementar la eficiencia de los sistemas personales por medio de instrumentos de medida del desempeño del sistema, etc.; y 2) asegurar la integridad del programa mediante programas de mantenimiento y seguridad, convertir programas de un lenguaje a otro, monitorear el uso de la máquina, etcétera.

${ }^{13}$ Son programas que permiten a los usuarios recuperar, organizar, manejar y manipular datos y bases de datos. Incluye todos los sistemas de administración de bases de datos, programas para toma de decisiones e información ejecutiva, hojas de cálculo, incluyendo Lotus 1-2-3 y sus imitaciones, etcétera.

${ }^{14}$ Incluye programas diseñados para proporcionar soluciones en paquete a problemas específicos propios de la administración o de los negocios. Específicamente, son programas que contribuyen a realizar funciones de contabilidad, administración de recursos humanos, nóminas, proyectos de inversión y procesamiento de palabras, así como actividades bancarias y financieras, manufactureras, cuidados de la salud, exploración de gas y petróleo, etcétera.

${ }^{15}$ Se refiere a la reparación y sustitución de componentes de hardware (incluyendo equipo de comunicación de datos, pero no de terminales específicas de la industria) y otros servicios proporcionados por el personal de mantenimiento de un solo vendedor, así como actividades de soporte de software no sujeto a licencia (soporte de teléfono, ayuda para el abastecimiento de escritorio, etc.).

${ }^{16}$ Se refieren a la prestación de servicios sobre una base contractual o de clientes para el desarrollo de sistemas y software; diseño, integración e instalación de sistemas relativos a educación y capacitación; facilidades de administración y servicios de consultoría sobre tecnologías de la información; etcétera. 
las del mercado nacional. Por lo tanto, se espera que la participación de los servicios profesionales se duplique. Entre las razones que explican esta tendencia se encuentra la mayor importancia que adquiere el diseño de sistemas y de programas más adecuados a las necesidades específicas del usuario, así como la urgente necesidad de personal capacitado que se tiene para el manejo de sistemas de computación. Estas tendencias son claramente consistentes con la dinámica del mercado mundial.

\subsection{Ciclo de vida del producto y precios}

La ITI se caracteriza también por una constante innovación tecnológica manifestada, entre otros factores, en la introducción de nuevos productos. La intensa competencia entre las empresas líderes obliga a mejorar continuamente el producto ofrecido, logrando innovaciones en su potencia, velocidad y versatilidad. Esta tendencia se traduce en la introducción constante de nuevos productos y/o en el perfeccionamiento de los productos existentes. Esta característica provoca que el ciclo de vida de algunos productos o de algunos componentes claves sea muy corto y que existan reducciones permanentes de los precios.

En una industria con economías a escala y competencia no perfecta (monopólica), la innovación permanente es condición para que los países y/o las empresas mantengan su ventaja en la medida que la tecnología de los productos modernos se difunde por efecto de las externalidades de las actividades de IyD, y por su carácter de bien público. El monopolio de la nueva tecnología, aunque temporal, les permite obtener ingresos extraordinarios que facilitan la cobertura de los gastos desembolsados en Iyd (Krugman, 1989 y 1990b; Varian, 1992). En la ITI, la introducción constante de nuevos productos, más potentes, versátiles, confiables y baratos, es la tónica desde su aparición. ${ }^{17}$ Todo el tiempo aparecen productos destinados a mercados particulares que tienen el objetivo de aprovechar las economías de escala dinámicas y de alcance. ${ }^{18}$

\footnotetext{
${ }^{17}$ En realidad, esta tendencia se inició con la introducción de la minicomputadora a fines de los años cincuenta por parte de la Digital Equipment Corp. (Mejía, 1998b).

${ }^{18}$ Por ejemplo: a mediados de 1996 la IBM anunció la introducción de una nueva familia de computadoras personales, la PC-100 y la actualización de su familia PC-300 (para la oficina pequeña y el hogar). HP, por su parte, presentó dos nuevos modelos de computadoras (Vectra XU y Vectra VT), así como de Omnibook, impresoras blanco y negro, impresoras láser y lectores ópticos (scanners) a color. Además, Apple introdujo
} 
La introducción permanente de nuevos productos se traduce de inmediato en la obsolescencia acelerada de los modelos existentes. El cuadro 3 muestra información respecto a la composición del mercado de PC por tipo de microprocesador. Se observa que después de que en 1981 los procesadores 8088/86 dominaban ampliamente el mercado, en 1994 prácticamente habían desaparecido. Aunque en menor grado, algo parecido ocurre con los procesadores 80286 y 80386 SX/DX. Estos productos han durado en el mercado diez, seis y siete años, respectivamente, y tal vez sólo los 80386SX tengan un par de años más de vida. En tan sólo cinco años los procesadores 80486 alcanzaron más de cuatro quintas partes del mercado, en especial los del tipo SX, seguidos por los DX2. Pero detrás de ellos vienen los Pentium ${ }^{19}$ a pasos agigantados, que en 1994 apenas representaban poco más de 1 por ciento.

Entre los factores determinantes de esta dinámica se encuentran los requerimientos del software, cuyas aplicaciones, cada vez más complejas, exigen mayor capacidad y potencia. La integración de los sistemas multimedia y la fusión de las telecomunicaciones con la informática, presionan a los productores en la misma dirección. ${ }^{20}$ Como se apuntó en el capítulo anterior, la dinámica del software determinará en buena medida la dinámica general de esta industria.

Los precios promedio de las PC, calculados en dólares, por su parte, muestran una reducción acelerada. La situación se torna menos grave a partir de la apertura comercial de 1985, sugiriendo que el ajuste a los patrones internacionales se había conseguido poco antes. Después de 1991 se observa otra caída resultante de la reducción de los gastos en informática de la administración pública y la apertura comercial, cuyo objetivo era obtener bienes y servicios informáticos a precios competitivos. Así, de 1981 a 1994 los precios promedio decrecieron de 5079 dólares a 1960 , es decir, disminuyeron en $61 \%$ a una tasa media anual de $7 \%{ }^{21}$ (véase gráfica 6).

también a mitad de año cuatro computadoras (modelos 7200, 7600, 8500 y 9500), un lector óptico (dirigido a oficinas caseras) y dos impresoras (para la empresa pequeña y el hogar) (El Financiero, 1996a, 1996b y 1996c).

${ }^{19}$ Recientemente, Select ha estimado que en el primer trimestre de 1996, 48\% de las PC vendidas incluían un microprocesador Pentium (El Financiero, 1996d).

${ }^{20}$ Además del factor cualitativo, las presiones cuantitativas en ese sentido crecen con rapidez. Según estimaciones de Select (El Financiero, 1996d) para el primer trimestre de 1996, 25\% de las computadoras vendidas incluía equipo multimedia.

${ }^{21}$ Actualmente las nuevas computadoras, con microprocesadores Pentium, multimedia, memoria en RAM y en disco duro incrementadas, fluctúan entre 2000 y 2400 
Cuadro 3

México: Indicadores de empresas líderes, IBM y HP, 1989-1994

\begin{tabular}{|c|c|c|c|c|c|c|}
\hline \multirow{2}{*}{ Empresa } & \multicolumn{5}{|c|}{ Años } & \multirow[b]{2}{*}{1994} \\
\hline & 1989 & 1990 & 1991 & 1992 & 1993 & \\
\hline \multicolumn{7}{|l|}{ IBM de México S. A. } \\
\hline $\begin{array}{l}\text { Exportaciones }(\mathrm{X}) \\
\text { Importaciones }(\mathrm{M}) \text { * }\end{array}$ & 37781.30 & 423768.39 & 437609.54 & 390577.17 & 570869.97 & 902370.63 \\
\hline Ventas totales $(\mathrm{V})$ * & $712057.2 \overline{3}$ & $823671.5 \overline{0}$ & $1034931.9 \overline{5}$ & $1026805.0 \overline{6}$ & $51070882.8 \overline{3}$ & $1407538.7 \overline{4}$ \\
\hline Ventas domésticas (D) * & 336275.94 & 399903.11 & 597322.41 & 636227.89 & 500012.86 & 505168.11 \\
\hline Activos (A) * & 304669.62 & 340138.11 & 426146.25 & 618240.64 & 603793.87 & 729278.27 \\
\hline Personal ocupado $(\mathrm{T})$ ** & 1866.00 & 2026.00 & 2145.00 & 2156.00 & 1636.00 & 1674.00 \\
\hline $\mathrm{X} / \mathrm{V}$ & 0.53 & 0.51 & 0.42 & 0.38 & 0.53 & 0.64 \\
\hline $\mathrm{M} / \mathrm{V}$ & ND & ND & ND & ND & ND & ND \\
\hline $\mathrm{X} / \mathrm{M}$ & ND & ND & ND & ND & ND & ND \\
\hline $\mathrm{X} / \mathrm{A}$ & 1.23 & 1.25 & 1.03 & 0.63 & 0.95 & 1.24 \\
\hline $\mathrm{M} / \mathrm{A}$ & ND & ND & ND & ND & ND & ND \\
\hline $\mathrm{V} / \mathrm{T}$ & 381.60 & 406.55 & 482.49 & 476.25 & 654.57 & 840.82 \\
\hline $\mathrm{D} / \mathrm{H}$ & 0.50 & 0.44 & 0.45 & 0.40 & 0.31 & 0.27 \\
\hline $\mathrm{D} / \mathrm{G}$ & 0.35 & 0.29 & 0.29 & 0.25 & 0.17 & 0.15 \\
\hline \multicolumn{7}{|l|}{ HP de México S. A. de C. V. } \\
\hline Exportaciones $(\mathrm{X}) *$ & 99040.01 & 133419.06 & 67441.61 & 66570.03 & 79161.20 & 85817.22 \\
\hline Importaciones $(\mathrm{M})$ * & 108099.08 & 100580.62 & 201063.28 & 237850.35 & 271005.27 & 357347.46 \\
\hline Ventas totales $(\mathrm{V})$ * & 211744.51 & 202185.42 & 273647.86 & 320935.79 & 468714.17 & 589803.70 \\
\hline Ventas domésticas (D) * & 112704.50 & 68766.36 & 206206.26 & 254365.76 & 389552.96 & 503986.48 \\
\hline Activo (A) * & 116154.70 & 134656.75 & 147077.57 & 182047.98 & 259981.68 & 396651.68 \\
\hline Personal ocupado $(\mathrm{T})$ ** & 758.00 & 920.00 & 1024.00 & 938.00 & 926.00 & 969.00 \\
\hline $\mathrm{X} / \mathrm{V}$ & 0.47 & 0.66 & 0.25 & 0.21 & 0.18 & 0.15 \\
\hline $\mathrm{M} / \mathrm{V}$ & 0.51 & 0.50 & 0.73 & 0.74 & 0.62 & 0.61 \\
\hline $\mathrm{M} / \mathrm{D}$ & 0.96 & 1.46 & 0.98 & 0.94 & 0.70 & 0.71 \\
\hline $\mathrm{X} / \mathrm{M}$ & 0.92 & 1.33 & 0.34 & 0.28 & 0.29 & 0.24 \\
\hline $\mathrm{X} / \mathrm{A}$ & 0.85 & 0.99 & 0.46 & 0.37 & 0.33 & 0.23 \\
\hline $\mathrm{M} / \mathrm{A}$ & 0.93 & 0.75 & 1.37 & 1.31 & 1.12 & 0.97 \\
\hline $\mathrm{V} / \mathrm{T}$ & 279.35 & 219.77 & 267.23 & 342.15 & 506.17 & 608.67 \\
\hline $\mathrm{D} / \mathrm{H}$ & 0.17 & 0.08 & 0.16 & 0.16 & 0.24 & 0.27 \\
\hline $\mathrm{D} / \mathrm{G}$ & 0.12 & 0.05 & 0.10 & 0.10 & 0.14 & 0.15 \\
\hline Total de harware $(\mathrm{H})$ & 672500.00 & 915100.00 & 1327600.00 & 1572800.00 & 1605100.00 & 1873500.00 \\
\hline Total del mercado $(\mathrm{G})$ & 971700.00 & 1386100.00 & 2067700.00 & 2581500.00 & 2858000.00 & 3280700.00 \\
\hline \multicolumn{7}{|l|}{ * Miles de dólares } \\
\hline$*$ Personas & & & & & & \\
\hline
\end{tabular}

Fuente: IDC-Select y Expansión, y cálculos propios.

En resumen, el mercado mexicano de computadoras muestra un comportamiento muy dinámico. El rubro de equipo sigue siendo el más importante, pero con una participación creciente de los segmentos de programas y servicios, destacando en el primer caso el software de soluciones aplicativas y en el segundo, los servicios profesionales. ${ }^{22}$ Pese a que se observa un crecimiento considerable en el número de computadoras promedio en la economía, nos encontramos muy lejos de los estándares interna-

dólares. Sin embargo, la Apple ha informado que lanzará al mercado un computadora de alrededor de 1000 dólares (El Financiero, 1996e).

${ }^{22}$ El crecimiento del mercado mexicano también se puede medir conforme el número de computadoras por cada mil habitantes: 0.141 en 1982, 3.2 en 1986, 8.6 en 1990, y 22 en 1994. El dato de 1982 se tomó de SELA (1985), cuadro 9; los de 1986 y 1990, de INEGI (1992:4); y el de 1994, de Poder Ejecutivo Federal (1996:128). 
cionales con respecto a computadoras por habitante y al nivel de conocimiento en el uso de computadoras. ${ }^{23}$ Sin embargo, precisamente estas estadísticas muestran la potencialidad de la demanda. Así mismo, ocurre una permanente introducción de equipos más poderosos, versátiles y baratos que satisfacen una demanda cada vez más diferenciada y reducen el ciclo de vida de los productos, lo cual evidencia un elevado dinamismo tecnológico y un régimen de competencia basado en la diferenciación del producto.

Gráfica 6

Precios promedio de las computadoras personales, 1980-1994 (dólares)

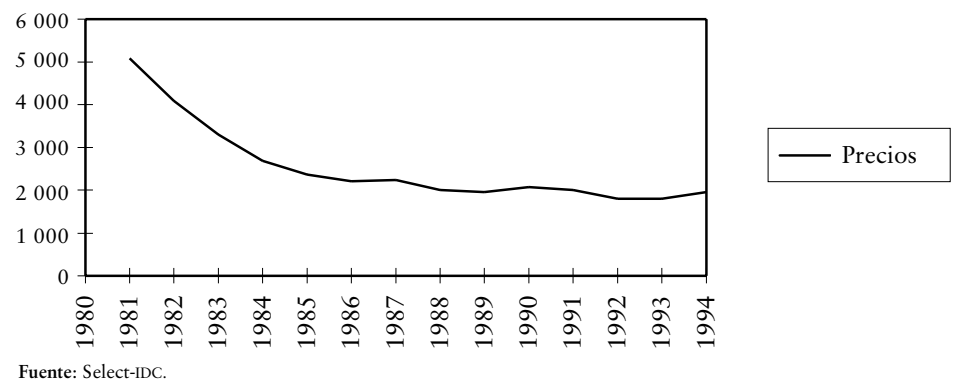

\section{Estructura del mercado}

La industria mundial de tecnologías de la información se puede caracterizar, en general, como un oligopolio inestable que se aproxima en gran medida a una industria con competencia monopólica, aunque con importantes diferencias entre sus segmentos constitutivos. Así, se aprecia un relajamiento en el mercado de los sistemas personales y el fortalecimiento del oligopolio de los sistemas de mayor tamaño. Entre las razones que explican tal patrón se encuentran la naturaleza de la demanda que exige sistemas versátiles, poderosos y, principalmente, de precio accesible; la naturaleza de la tecnología de los equipos pequeños, que por

${ }^{23}$ Los Estados Unidos encabezan el registro de computadoras por cada mil habitantes con casi 300 máquinas. Por otro lado, en México solamente $5.6 \%$ de la población urbana sabe operar una computadora (Poder Ejecutivo Federal, 1996:129). 
ser relativamente sencilla se convierte de modo inminente en un bien público, con importantes externalidades; y el potencial del mercado de este tipo de equipo que atrae más a las empresas hacia este segmento, en el que la diferenciación del producto y la integración de paquetes de venta constituyen las estrategias de competencia más empleadas, y donde no hay necesidad de enfrentarse a productores gigantes (Ernst y O’Connor, 1992; Mejía, 1998b). Sin embargo (como han apuntado los primeros autores), el control del mercado se efectúa cada vez menos en la etapa de obtención del producto final, y más en la producción de insumos claves o en el control de las cadenas de abastecedores y distribuidores. En ese sentido, el surgimiento de barreras a la entrada ha retrocedido hacia las etapas iniciales de la cadena productiva (Ernst y O'Connor, 1992). Por ejemplo, en el caso de la producción de las PC, la importancia de Intel en el mercado de microprocesadores es muy clara. En el mercado mexicano, los productores de computadoras personales que en 1994 incorporaban productos de Intel representaron 96\% del valor de los embarques $^{24}$ (véase cuadro 3).

En un país menos desarrollado las barreras a la entrada que enfrentan los productores locales son mayores, pues a la brecha tecnológica existente se suma la ausencia de las externalidades propias de los conglomerados industriales de los países desarrollados. En consecuencia, las ventajas de los últimos en el desarrollo de industrias de alta tecnología resultan acumulativas, tal como lo ha argumentado Krugman (1990a, 1990b). Es bastante plausible argumentar que en el caso de México, las barreras a la entrada, sobre todo las de carácter tecnológico y de acceso a las cadenas de abastecedores y comercializadores, ${ }^{25}$ han determinado la aparición de un mercado altamente oligopolizado. Son ET las que rigen los distintos segmentos. Éstas desarrollan principalmente funciones de comercialización, ensamblaje y fabricación de equipo -en ese orden de importancia-, aprovechando las ventajas brindadas por la economía mexicana, la cual es empleada como plataforma de exportación, por un lado, y para satisfacer el creciente mercado doméstico, por otro. La ilustración de casos específicos mostrará de manera más detallada estas características. cios.

${ }^{24}$ Es el total de recursos pagados a los vendedores por sistemas, software y servi-

${ }^{25}$ Warman y Miller $(1988: 15)$ consideran que las barreras de umbral en la planta nunca fueron insalvables. 
Entre las 500 empresas más importantes de México, lista publicada anualmente por la revista Expansión, se encuentran dos dedicadas a la producción, al ensamble y comercialización de equipo de cómputo: la IBM y la Hewlett-Packard (HP), ambas estadounidenses. En el cuadro 3 se presentan las características más importantes de ambas. En el primer caso, los indicadores de ventas -ventas totales, V; ventas domésticas, D; y exportaciones, $\mathrm{X}-$ muestran un crecimiento permanente entre 1989 y 1994, excepto en 1992 y 1993. Algo similar ocurre con los indicadores correspondientes a la HP, sólo que los periodos de decrecimiento son 1990 para los primeros dos rubros y 1991-1992 para las exportaciones. En esos años, recordemos, ocurrieron dos eventos de singular importancia para el desempeño del mercado: la eliminación de los permisos de importación de equipo, y la reducción del gasto en informática del sector público. ${ }^{26}$ Sin embargo, la posibilidad y necesidad de exportar sus productos, dada la contracción del mercado doméstico, ha permitido a estas empresas mantener su crecimiento de largo plazo. Una medida indirecta de este hecho se aprecia en el comportamiento de sus activos: los activos (A) de la IBM y de la HP crecieron a una tasa de $24.4 \%$ y de 33.6\%, respectivamente, durante el periodo 1989-1994.

Por otro lado, el empleo $(\mathrm{T})$ empezó a descender desde principios de la década de los noventa. El crecimiento sostenido en la relación ventas totales a empleo, $\mathrm{V} / \mathrm{T}$, da cuenta de una mayor productividad del trabajo como una de las principales causas de la expansión de ambas empresas. Este patrón no es sorprendente en virtud de que aun cuando las actividades de ensamblaje son muy importantes en ambas empresas, como veremos más adelante, es perfectamente factible obtener economías a escala dinámicas y de alcance a consecuencia de la especialización de las plantas en ciertas líneas de productos, y de la comercialización de bienes importados vía comercio intrafirma.

Se debe destacar que, aunque las ventas totales de la IBM son dos veces y media las de la HP, la participación de las ventas domésticas de ambas en las ventas totales de hardware $(\mathrm{H})$ y en el total del mercado $(\mathrm{G})$ es similar debido a que las exportaciones de la primera son más de diez veces las de la última.

${ }^{26}$ En el siguiente inciso se efectúa un análisis de la política industrial implantada durante la mayor parte de los años ochenta para impulsar la producción de equipo de cómputo. 


\section{Cuadro 4}

México: Indicadores de actividad en la industria de computadoras, 1981-1994*

(millones de dólares)

\begin{tabular}{|ccccc|}
\hline Año & $\begin{array}{c}\text { Producción } \\
\mathrm{P}\end{array}$ & $\begin{array}{c}\text { Inversión } \\
\text { I }\end{array}$ & P/I & $\begin{array}{c}\text { Personal } \\
\text { ocupado } * *\end{array}$ \\
\hline 1982 & 59 & & & 1600 \\
1983 & 99 & 20 & 0.20 & 2754 \\
1984 & 135 & 19.5 & 0.14 & 3898 \\
1985 & 145 & 19.4 & 0.13 & 5041 \\
1986 & 135 & 2.5 & 0.02 & 5160 \\
1987 & 314 & 10.5 & 0.03 & 5763 \\
1988 & 620 & 13 & 0.02 & 6405 \\
1989 & 988 & 19 & 0.02 & 6510 \\
1990 & 1100 & 7 & 0.01 & 2743 \\
1991 & 1350 & 4 & 0.00 & 2411 \\
1992 & 1370 & 8 & 0.01 & 2501 \\
1993 & 1575 & 13 & 0.01 & 2254 \\
1994 & 1714 & & & 2492 \\
$*$ 1993 y 1994 son datos preliminares & & \\
$*$ Personas & & & \\
\hline
\end{tabular}

Fuente: SECOFI, con datos del Sistema de Información Comercial de México y el Sistema de Cuentas Nacionales, en CANIECE (1994), Serra (1994:13) y Borja (1995:195 y 200).

Para reiterarlo, la ITI se caracteriza por la convivencia de dos tendencias aparentemente contradictorias. Por un lado, existe una importante concentración de mercado, mientras que por el otro, hay una competencia creciente dentro de sus distintos segmentos, sobre todo en aquellos en que la tecnología se homologa más rápido. De acuerdo con la información del cuadro 3, al parecer existe mayor competencia dentro del mercado, puesto que ha habido una reducción en la participación de la IBM en el total de hardware y en el total del mercado. En el primer caso, D/ H disminuyó de 50\% en 1989 a 30\% en 1994, en tanto que D/G bajó de 30 a 20\%. Esta tendencia se complementó con el fortalecimiento de la posición de la HP. Entre 1989 y 1994 D/H y D/G aumentaron de 20 a $30 \%$ y de 10 a 20\%, respectivamente. Sin embargo, a pesar de que ha habido una ligera reducción en uno de los casos, la suma de las participaciones de ambas empresas en 
los totales mencionados se ha mantenido más o menos estable. Así, la suma de sus participaciones en $\mathrm{H}$ en 1989 era de $70 \%$ mientras que en 1994 equivalía a 60\%. Por su parte, sus participaciones en $\mathrm{G}$ en el primero y en el último año eran de $40 \%$. Al parecer, las tendencias que caracterizan al mercado mundial se reproducen en gran medida en el mercado mexicano.

La concentración de mercado respecto al producto puede presentar diferencias importantes con relación a las empresas líderes. De ocurrir, tal situación podría reflejar en algunos casos la especialización de las empresas en ciertos segmentos del mercado para aprovechar las economías de escala, mientras que en otros podría estar mostrando la tendencia de las empresas a aprovechar al máximo las economías de alcance. Así mismo, los cambios en las posiciones de liderazgo evidencian la elevada movilidad de las empresas y la inestabilidad propia del mercado. Algunas inferencias pueden obtenerse de la información sobre las empresas líderes en los mercados de dos productos para los que se dispone de información.

El primer caso es el de las PC. Su mercado ha experimentado una significativa recomposición. En 1988 era una empresa mexicana la que encabezaba el mercado, al amparo de la política de fomento, y superaba a las transnacionales: Printaform tenía una participación en el mercado mexicano (36\%) superior a la de gigantes mundiales de la talla de IвM (más de dos veces y media su participación en el mercado) y HP (más de tres veces su participación en el mercado). Además, entre las primeras diez aparecían empresas nacionales como Flujo de Datos, Electrón y Gama (véase gráfica 7).

\section{Gráfica 7}

México: Principales empresas vendedoras de computadoras personales, 1988

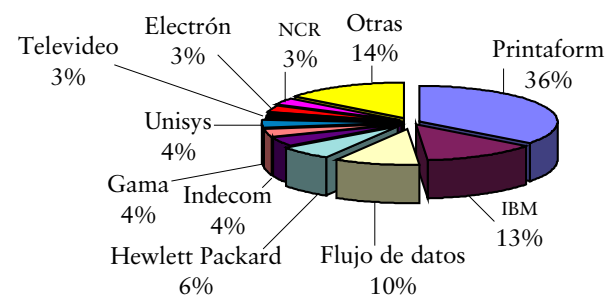

Fuente: Microsoft Corp. 
Los cambios de política económica iniciados desde 1985 y culminados en 1994 con el Tratado de Libre Comercio de América del Norte (TLCAN), cambiaron en forma significativa y definitiva las cosas para el primer semestre de 1995. Para entonces, muchas empresas mexicanas habían salido de la ITI. El mercado estaba claramente dominado por la empresa comercializadora Acer, que triplicaba el volumen de ventas de sus cuatro siguientes perseguidores (HP, Compaq, Digital e IBM). Además, las tres empresas más grandes absorbían alrededor de 75\% del mercado y entre las diez más grandes la única mexicana era Printaform, con un modesto $2.9 \%$ del mercado (gráfica 8 ). Dado el predominio mundial de empresas estadounidenses como Compaq o IBM, resulta curioso que una empresa taiwanesa domine este segmento en México. La razón puede encontrarse en el carácter público de la tecnología empleada en la producción de este tipo de bienes y en la especialización que Acer ha desarrollado, permitiéndole explotar las economías de escala dinámicas y estáticas. Además, esta empresa ha usado la continua reducción de precios como estrategia principal de competencia.

\section{Gráfica 8}

México: Principales empresas vendedoras de computadoras personales, 1995*

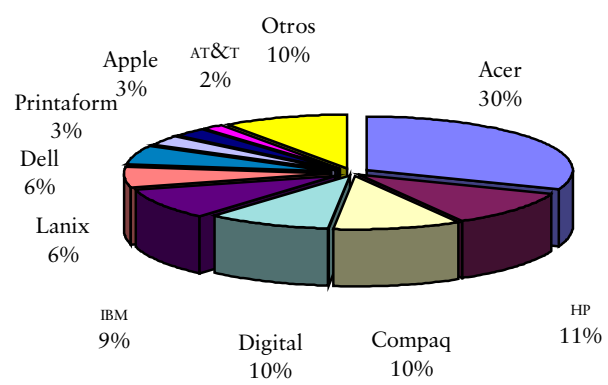

* Primer semestre de 1995 
El perfil descrito del mercado durante 1995 se aproxima en gran medida al internacional en el sentido de que las empresas que se han especializado en la producción de microcomputadoras son las que dominan este segmento en México. Como lo muestra Mejía Reyes (1998b), HP, Compaq, DEC e IBM, que ocuparon los lugares 10, 1, 9 y 2 dentro de este segmento en el mercado mundial en 1994, son líderes en el mercado mexicano.

Una apreciación más completa de los cambios en la estructura del mercado y de la dinámica de la competencia dentro de este segmento, se puede lograr mediante el uso de los índices de concentración que satisfacen las propiedades requeridas (Tirole, 1988:223). Para lo primero utilizaremos el Índice de Herfindahl (IH), el cual es muy empleado en la literatura de la organización industrial para medir la concentración de la producción. El IH puede variar entre un valor mínimo igual a $1 / \mathrm{n}$-correspondiente a una situación en la que todas las empresas tienen la misma participación de mercado-, y uno máximo de 1 -correspondiente a una situación de monopolio. Cualquier fusión de empresas se refleja en este caso en un incremento en el valor de $H$. Se define como

$$
I H=\sum_{i=1}^{m} \alpha_{i}^{2}
$$

donde

$$
\alpha_{i}=\frac{q_{i}}{Q} \operatorname{con} \sum_{i=1}^{n} \alpha_{i}=1
$$

donde a su vez $q_{i}$ representa la participación de la $i$-ésima empresa en el total de la industria, y $Q$ es el total de la industria.

Para medir la evolución de la competencia se utiliza el Índice de Dominación $(P)$ propuesto por García Alba Iduñate (1994), el cual no penaliza cualquier fusión, sino que lo hace en función de la estructura particular del mercado y del tamaño relativo de las empresas fusionantes: la fusión de dos empresas pequeñas no aumenta el valor del índice, pero sí la de dos grandes; en el primer caso el índice puede reducirse indicando una mayor competencia entre las empresas (las empresas fusionadas tienen un tamaño mayor que les permite competir en mejores condiciones con las empresas más grandes), en tanto que en el segundo, el incremento en el valor de $P$ indicaría una menor competencia -pues las empresas fusionadas tendrían mayor poder de mercado-. Se 
puede interpretar como un índice de concentración de la concentración, y se define, de manera análoga al IH, como

$$
P=\sum_{i=1}^{n} h_{i}^{2}
$$

donde

$$
h_{i}=\frac{\alpha_{i}^{2}}{H}
$$

Estos índices se aplicaron a los datos del mercado de PC de 1988 y 1995 para las primeras diez empresas. En 1988 IH y P fueron iguales a 0.16 y 0.60 , y en 1995 , a 0.14 y 0.45 , respectivamente. Por lo tanto, puede considerarse que, al igual que la tendencia en el mercado internacional, hubo una reducción en la concentración de las ventas por empresa, y un incremento en la competencia entre los participantes, lo cual también puede observarse en el crecimiento en la participación relativa de las empresas que siguen a los tres primeros lugares, así como en la reducción de la participación del líder para cada uno de los años.

Por otra parte, la ampliación del uso de redes ha desarrollado un sector más, el de los servidores. Las ET, como parte de su estrategia para no perder el liderazgo, se han desplazado hacia la producción de sistemas más pequeños (Mejía, 1998b), y dada la potencialidad de las redes han penetrado desde hace tiempo en el rubro de servidores. En este caso no podemos establecer un análisis como en el anterior, debido a que tenemos información únicamente para un año y a que sólo son cinco las empresas que dominan este segmento del mercado. Este rubro está altamente concentrado y es dominado por tres empresas, Acer Altos, Compaq y HP, las cuales concentran más de cuatro quintas partes del mercado (véase gráfica 9).

En resumen, el mercado mexicano presenta una dinámica similar a la del mercado internacional: existe una importante concentración del mercado acompañada por una creciente competencia. Además, el mercado se encuentra dominado por un pequeño grupo de ET, las cuales se han especializado en la producción de ciertas líneas de productos. Así, luego de que durante la mayor parte de la década pasada, el mercado mexicano de las PC estuvo dominado por una empresa mexicana, hoy día las empresas del país desempeñan un papel marginal. En la conformación de esta estructura de mercado han intervenido múltiples factores, tales como ausencia de actividades de IyD, falta de iniciativa 
empresarial, inconsistencia de las políticas de fomento, inexistencia de una base de capital humano, etc. La dinámica de la inversión y de la producción, así como el tipo de bienes producidos son factores analizados en el resto de este artículo.

\author{
Gráfica 9 \\ México: Mercado de servidores, 1995 \\ (estructura porcentual)
}

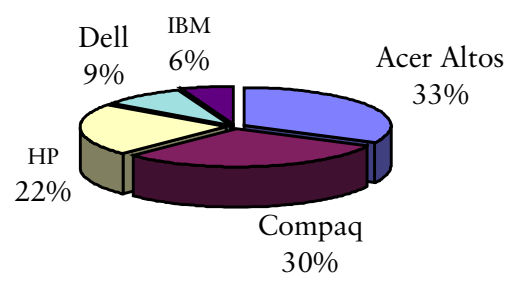

Fuente: Select-IDC.

\title{
4. Evolución de la inversión y de la producción en la industria de computadoras
}

En esta sección se analiza el origen y la evolución de la producción de equipo de cómputo en México a partir de la década de los ochenta. Se examinan los efectos de los cambios en la política para esta industria en términos de inversión y producción, y se presenta información para entender por qué las empresas mexicanas fueron forzadas a salir del mercado.

La producción de computadoras se inició en México a raíz de la implantación del Programa de fomento para la manufactura de sistemas electrónicos de cómputo, sus módulos principales y sus equipos periféricos, propuesto por la entonces Secretaría del Patrimonio y Fomento Industrial (SEPAFIN, 1981). En un marco propio de la política de sustitución de importaciones, se definieron los siguientes objetivos del programa: producir internamente computadoras personales y minicomputadoras; crear una industria nacional de partes y componentes para la producción de computadoras; generar capacidad de exportación; y lograr la autonomía tecnológica. Los objetivos se cumplirían mediante el diseño 
de políticas específicas proteccionistas y temporales en aspectos de comercio exterior, inversión extranjera, IyD y subsidios e impuestos. La crisis económica de 1982 y los cambios en la orientación de la política económica eliminaron de hecho esta política de fomento. Se cambio así a un marco en el que el mercado sería lo que determinaría las decisiones relativas a las ті. ${ }^{27}$

Recientemente ha aparecido el Programa de Desarrollo Informático dentro del Plan Nacional de Desarrollo 1995-2000. El objetivo general es impulsar el uso de la informática en los distintos sectores de la economía como medio para mejorar la competitividad. De hecho, no constituye un programa de fomento directo a esta industria nacional, y la forma más importante de estímulo es mediante el otorgamiento de incentivos a la demanda para acrecentar su consumo. Desde esa perspectiva, los apoyos del lado de la oferta son mínimos. ${ }^{28}$

El programa original de 1981 era completo. Había consistencia entre los objetivos y los medios tendientes a la creación de una planta para la producción doméstica de equipo y de componentes, así como la obtención de la autonomía tecnológica, aunque ninguno de ellos era fácil de conseguir y, tal vez, el último enfrentaría difíciles obstáculos. Sin embargo, el objetivo de crear una base exportadora firme era inconsistente, puesto que las medidas tomadas creaban de hecho un mercado cautivo en el que, en principio, se tendrían que presentar precios superiores a los internacionales, restándole competitividad a la industria. ${ }^{29}$ Sin embargo, la consistencia que pudo haber existido al principio se diluyó en mayor medida con las transformaciones operadas a partir de 1985. La apertura comercial y la liberalización de la inversión extranjera en materia de producción de PC, implicó la eliminación de la reserva de mercado y el desplazamiento de las empresas mexicanas de uno de los sectores más dinámicos del mercado, en el que además las barreras a la entrada eran menores. Así mismo, la apertura comercial era incompatible con la exigencia de requisitos de integración nacional y de coeficientes de exportación/importación, debido a que se dejaba prácticamente abierta

\footnotetext{
${ }^{27}$ Véase Mejía Reyes, 1997 para una revisión más completa de la naturaleza de la política de fomento y de las transformaciones que le siguieron después de 1982.

${ }^{28}$ Se propone el estímulo a las actividades de investigación mediante apoyos fiscales y el fortalecimiento de los programas de estudios en informática, con el objeto de avanzar en la conformación de una base amplia que permita la explotación eficiente de la infraestructura existente (Poder Ejecutivo Federal, 1996:65-69 y 73-77).

${ }^{29}$ Véase Borja, 1995, pp. 103-104.
} 
la puerta a las importaciones de partes y componentes. El objetivo de lograr la autonomía tecnológica quedó así relegado.

Las nuevas disposiciones en materia de informática impulsarán sin duda el uso de los bienes y servicios informáticos, con lo que crecerá la demanda, pero sin duda ésta será satisfecha con importaciones en virtud de que no se perciben medidas que apoyen decididamente la producción doméstica.

La eliminación de la política original, en especial la liberalización del comercio y de la IE (aunque en el fondo está la incapacidad de las empresas nacionales para ser competitivas en un ambiente no protegido), ha determinado la naturaleza de la industria en el momento actual: una industria dedicada prácticamente al ensamblaje de equipo, dominada por algunas ET, con mínimos o nulos gastos en IyD, incapaces de satisfacer la dinámica demanda doméstica, lo que en consecuencia genera un déficit comercial creciente. ${ }^{30}$

A partir de su implantación, el programa tuvo significativos efectos en la producción doméstica de equipo, pues permitió la transformación de distribuidores en productores, quienes paulatinamente definieron su propia red de distribución. Un dato interesante es que durante los primeros cinco años de la década de los ochenta, se instalaron todas las empresas (menos una) que hasta 1993 fabricaban computadoras en México y todas las que exportaban, aunque muchas otras habían cerrado (Warman y Miller, 1988:11-15; Warman, 1994:402-406).

Los resultados fueron relativamente rápidos una vez que se inició el programa, y desde el principio las ET desempeñaron un papel central. ${ }^{31}$ En el cuadro 4 se observa que la inversión empezó a niveles altos -para cubrir los requerimientos de las barreras de umbral-, aunque siempre, a excepción de 1987, presentó tasas negativas cada vez mayores. Su evolución estuvo condicionada por el comportamiento general de la economía y, dada su importancia en el mercado, por la política gubernamental de gasto en productos de computación, así como por los cambios que subsecuentemente se introducían restando consistencia al programa de fomento. Así, la inversión estuvo restringida por la contracción del mercado y el ajuste fiscal hasta 1986. doras.

${ }^{30}$ Véase Mejía Reyes, 1997 para un análisis del comercio internacional de computa-

${ }^{31}$ Pronto se instalaron filiales de IBM, Wang, HP, Apple, Data General, NCR, Digital Equipment, Sperry -que posteriormente participó en la creación de Unisys- y Tandem (Warman y Miller, 1988). 


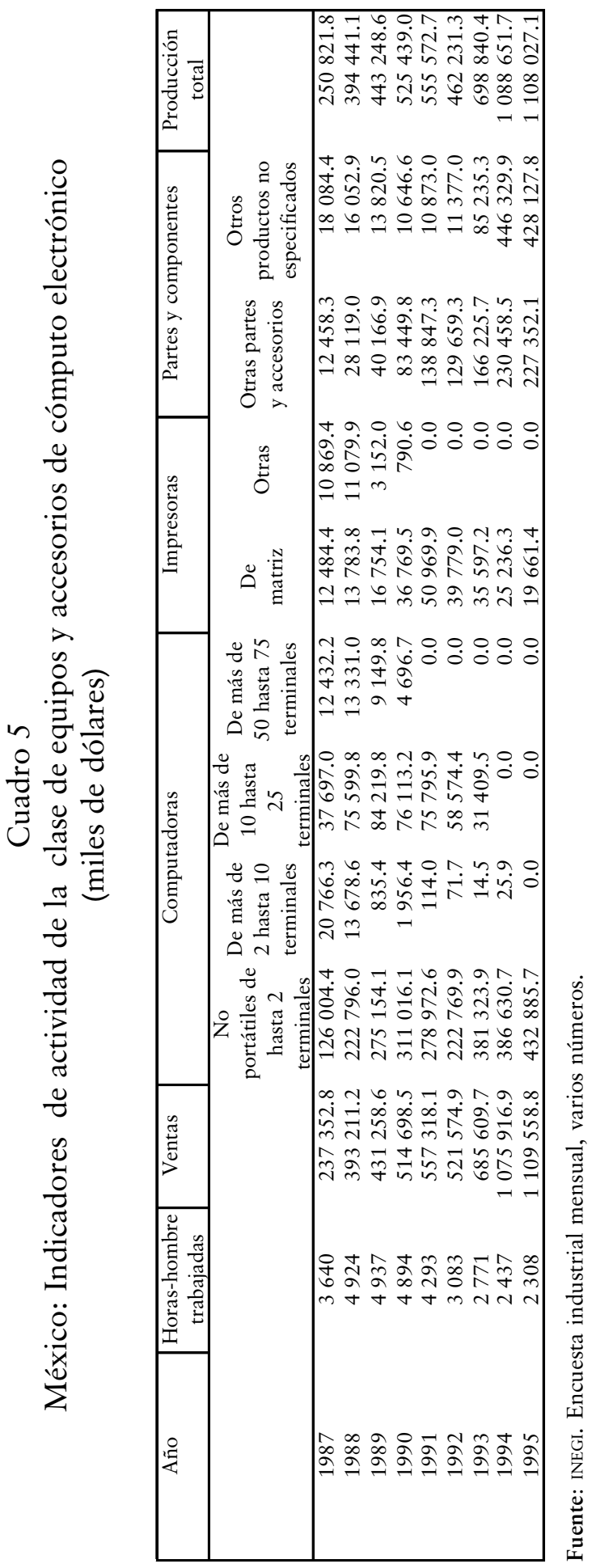


La liberalización comercial y de la IE iniciadas en 1985 fueron apropiadas, pues permitieron contrarrestar los efectos adversos, principalmente después de 1987, y entonces fue posible aumentar la producción interna (de PC) abasteciéndose con insumos importados. Sin embargo, después de 1990 la profusión de la liberalización comercial y el menor peso de la administración pública en el mercado, inhibieron aún más la inversión. La falta de competitividad de la industria nacional no soportó la competencia externa y las perspectivas de crecimiento se volvieron adversas. La producción tuvo un comportamiento similar al de la inversión, aun cuando la tendencia decreciente hacia el final del periodo fue menor. Consecuentemente, la razón inversión/producción disminuyó de manera acelerada y el empleo empezó a declinar a partir de $1990 .^{32}$

Por otra parte, la información presentada en el cuadro 5 nos permite conocer el perfil de la producción doméstica. ${ }^{33} \mathrm{Al}$ igual que el empleo, las horas-hombre trabajadas disminuyen después de 1990, mientras que las ventas y la producción total siguen un crecimiento estable, lo que refleja un incremento importante en la productividad del trabajo. La contracción del mercado doméstico sugiere también que una parte significativa del producto se canalizaba al mercado externo. La composición de la producción muestra, por su parte, la limitación de la producción a computadoras pequeñas, pues en 1995 se obtuvieron computadoras no portátiles de hasta dos terminales; algo parecido ocurrió con las impresoras ya que solamente se produjeron de matriz. El rubro que ha permitido el crecimiento total de la producción es el de otras partes y componentes.

Se observa entonces que la producción doméstica (no únicamente la mexicana) se ha limitado a la obtención de bienes que emplean tecnología estandarizada, de cuya producción los líderes mundiales se han desligado o bien han transferido hacia los países menos desarrollados las etapas menos complejas del proceso productivo.

Los avances respecto al tipo de productos generados internamente dejan mucho que desear. En general, la producción de computadoras en México se ha caracterizado por un rezago tecnológico evidenciado en la producción de bienes cuya tecnología

\footnotetext{
${ }^{32}$ Una imagen similar se puede obtener a partir de la información censal presentada en Mejía Reyes, 1997.

${ }^{33}$ Los resultados pueden diferir con respecto a los presentados antes porque esta información es muestral.
} 


\section{Cuadro 6}

México: Empresas internacionales fabricantes de equipo de informática

\begin{tabular}{|c|c|c|c|}
\hline Empresa & Actividad & Mercado & Productos \\
\hline IBM & Fabricación IyD & Nacional y exterior & $\begin{array}{l}\text { Computadoras } \\
\text { Periféricos } \\
\text { Impresoras }\end{array}$ \\
\hline Hewlett-Packard & $\begin{array}{l}\text { Fabricación Diseño } \\
\text { IyD }\end{array}$ & Nacional y exterior & $\begin{array}{l}\text { Computadoras } \\
\text { Periféricos } \\
\text { Impresoras }\end{array}$ \\
\hline Digital Equipment & $\begin{array}{l}\text { Fabricación Diseño } \\
\text { IyD }\end{array}$ & Nacional y exterior & $\begin{array}{l}\text { Computadoras } \\
\text { personales } \\
\text { Mainframes }\end{array}$ \\
\hline Olivetti & Fabricación & Nacional y exterior & $\begin{array}{l}\text { Calculadoras } \\
\text { Máquinas de } \\
\text { escribir } \\
\text { Computadoras }\end{array}$ \\
\hline NCR & Fabricación & & $\begin{array}{l}\text { Estaciones de } \\
\text { trabajo } \\
\text { Redes locales }\end{array}$ \\
\hline
\end{tabular}

Fuente: "Directorio: conectividad, protección y distribuidores", Alta tecnología, mayo- junio de 1990; Servidigit, Perfil de Expositores, Feria Mexcom, 1991, enero de 1991; y Ramón Tirado Jiménez, "Telefonía y redes de telecomunicaciones. El caso de diez empresas en México", El economista mexicano, Colegio Nacional de Economistas, abril-junio de 1993; en Tirado Jiménez, 1994.

se ha homologado. Warman (1993:401) sugiere que la situación es resultado de la cultura empresarial sin el suficiente interés, en tanto que Tirado (1994) argumenta que una razón más es la escasez de una base firme de capital humano que permita tener condiciones para la innovación. Así mismo, es plausible considerar como causa adicional la ausencia de actividades de IyD, y con ello, la imposibilidad de aprovechar las externalidades de la generación de tecnología. Es muy probable que la "pérdida" de los avances cuando el programa de fomento devino uno en el que la competencia era frontal con los productores internacionales tradicionales, se deba a la ausencia de factores como éstos.

A la luz de la evolución de sectores específicos luego de la liberalización comercial, resulta cada vez más evidente que las políticas de fomento crearon y sostuvieron artificialmente sectores en los que no había posibilidades de crecer de manera sostenida en un ambiente no proteccionista. Krugman (1994) y Krug- 
man y Obstfeld (1988: caps. 9-11) presentan argumentos en los que demuestran lo inconveniente de impulsar industrias para las cuales no existe una base que dé una ventaja comparativa al país, o cuando no hay perspectivas de que tal base pueda desarrollarse en un periodo razonable. Por su parte, Romer (1991) acentúa la importancia del capital humano como condición para crecer y como factor central para una industria basada sobre todo en el conocimiento.

\section{Consideraciones finales}

El desempeño de la ITI en México durante el periodo 1980-1995, refleja la evolución de la tecnología en los mercados internacionales. El mercado nacional es un mercado activo en el que los servicios y el software son los rubros más dinámicos, aunque el renglón de equipo es el de mayor peso. Respecto al hardware se da un desplazamiento hacia los sistemas pequeños, como las PC y las estaciones de trabajo, debido a la tendencia del mercado a favorecer el uso de sistemas pequeños, versátiles y baratos. La introducción de nuevos equipos es constante, lo que incrementa la competencia, reduce los precios, y significa un corto ciclo de vida del producto. En los segmentos de programas y servicios destaca el software de soluciones aplicativas y los servicios profesionales. Este patrón de conducta es perfectamente congruente con la dinámica mundial.

La estructura del mercado para las PC y los servidores es muy concentrado y las ET que rigen en un segmento lo hacen también en el otro. Sin embargo, en el mercado de PC ha habido de 1988 a 1995 una reorganización completa en las posiciones. Después de que en el primer año era una empresa mexicana la que encabezaba el mercado y varias empresas nacionales se ubicaban entre las primeras diez, en el último año el mercado era controlado exclusivamente por ET, indicando que éstas aprovechan de mejor manera las economías de escala explotadas en diferentes partes del mundo, y que las empresas mexicanas tienen dificultades para salvar las barreras a la entrada propias de la industria sin apoyo gubernamental. Como consecuencia de las medidas de desregulación y liberalización comercial (aunque se sabe que en el fondo se encontraba la ineficiencia de las empresas mexicanas), la producción local se desaceleró a partir de 1990 y 
desde entonces se ha limitado a producir las computadoras e impresoras de tecnología estandarizada.

Las barreras a la entrada en todas las etapas de la producción y comercialización impiden la creación de empresas nacionales competitivas. En este sentido, la experiencia muestra que la política de impulso inicial fue parcialmente exitosa, pero que su discontinuidad e inconsistencia, combinadas con la falta de una base de capital humano y de iniciativa empresarial y capacidad innovadora, impidieron el fortalecimiento de una industria nacional productora de equipo de cómputo.

Las implicaciones de las recientes medidas en materia informática se traducirán en un mayor uso de ella en las distintas actividades económicas, $y$, por ende, en un incremento de la demanda. Sin embargo, la rigidez de la oferta conduce a pensar que la demanda será satisfecha con un incremento de las compras al exterior.

\section{Bibliografía}

Borja, Arturo (1995), El Estado y el desarrollo industrial. La política mexicana de cómputo en una perspectiva comparada, México, Centro de Investigación y Docencia Económicas-Porrúa.

Carliner, Geoffrey (1991), "Las políticas industriales para industrias emergentes”, en Paul R. Krugman (comp.), Una politica comercial estratégica para la nueva economía internacional, México, Fondo de Cultura Económica.

Coriat, Benjamín (1992), El taller y el robot, México, Siglo XXI.

Datamation (1995), “The Datamation 100”, Nueva York, varios números.

El Financiero (1996a), “IBM presenta nuevas familias de PCs en México”, México, D. F., 23 de julio, p. 9A.

— (1996b), "Anunció Hewlett-Packard nuevos productos para AL”, México, D. F., 18 de junio, p. 8A. 
- (1996c), "Anunció Apple nuevos productos e inauguró oficinas en México", México, D. F., 25 de junio, p. 9A.

— (1996d), "Select libera cifras del primer trimestre", México, D. F., 27 de junio, p. 17A.

— (1996e), "Apple Computer lanzará una PC cuyo precio será de aproximadamente siete mil 500 pesos", México, D. F., 30 de julio, p. 8A.

Ernst, Dieter y David O'Connor (1992), Competing in the Electronics Industry, París, Organización para la Cooperación y el Desarrollo Económico.

Expansión (1990-1995), "Las empresas más importantes de México", México, varios números.

Flamm, Kenneth (1987), Targeting the computer. Government support and international competition, Washington, The Brookings Institution.

García Alba Iduñate, Pascual (1994), "Un índice de dominación para el análisis de la estructura de los mercados", El Trimestre Económico, vol. LXI(3), núm. 243, julio-septiembre, pp. 499-524.

Grunwald, Joseph y Kenneth Flamm (1985), The Global Factory. Foreign Assembly in International Trade, Washington, The Brookings Institution.

Instituto Nacional de Estadística, Geografía e Informática (INEGI) (1992), La situación de la informática en México, MéxiCO, INEGI.

(1994), La informática en México, México, INEGI.

Krugman, Paul R. (1989), "Industrial Organization and International Trade", en Richard Schmalensee y Robert D. Willing (eds.), Handbook of Industrial Organization, vol. 2, Amsterdam, Elsevier Science Publishers B.V. 
_ (1990a), “Trade, accumulation and uneven development”, en George Thomas, Rethinking international trade, Cambridge, Mass., The MIT Press, pp. 93-105.

(1990b), "A model of innovation, technology transfer, and the world distribution income", en George Thomas, Rethinking international trade, Cambridge, Mass., The MIT Press, pp. 139-151.

_ (1994), "Competitiveness: A dangerous obsession”, Foreign Affairs, vol. 73, núm. 2, marzo-abril, pp. 28-44.

— y Maurice Obstfeld (1988), International economics. Theory and policy, Glenview, Illinois, Scott, Foresman and Company.

Mattar, Jorge y Claudia Schatán (1993), “El comercio intraindustrial e intrafirma México-Estados Unidos: autopartes, electrónicos y petroquímicos", Comercio Exterior, vol. 43, núm. 2, febrero, pp. 103-124, México, BANCOMEXT.

Mejía Reyes, Pablo (1997), Tecnología y competencia imperfecta en la industria mexicana de computadoras, Zinacantepec, México, El Colegio Mexiquense, Documento de Investigación 8 .

- (1998a), "Competencia y comercio internacional en la industria de computadoras", Ciencia Ergo Sum, vol. 5, núm. 2, julio, pp. 115-124, Toluca, UAEM.

_ (1998b), “Dinámica y competencia monopólica en la industria mundial de las tecnologías de la información", The Anábuac Journal, vol. 2, núm. 1, fall/winter, pp. 40-59, México, Universidad Anáhuac del Sur.

Poder Ejecutivo Federal (1996), Programa de Desarrollo Informático, 1995-2000, México, Gobierno de la República.

Romer, Paul (1991), “El cambio tecnológico endógeno”, El Trimestre Económico, vol. LVIII (3), núm. 231, julio-septiembre, pp. 441-480. 
Salinas Chávez, Antonio (1993a), "La industria de la computación: avances tecnológicos y desempeño reciente (Primera parte)", Comercio Exterior, vol. 43, núm. 3, marzo, pp. 270-275, México, BANCOMEXT.

(1993b), "La industria de la computación: avances tecnológicos y desempeño reciente (Segunda parte)", Comercio Exterior, vol. 43, núm. 3, mayo, pp. 507-512, México, BANCOMEXT.

Secretaría del Patrimonio y Fomento Industrial (SEPAFIN) (1981), Programa de fomento para la manufactura de sistemas electrónicos de cómputo, sus módulos principales y sus equipos periféricos, México, SEPAFIN.

SELA (1985), Estrategias de las empresas transnacionales en informática en el mercado mundial y en América Latina, MéxiCO, SELA.

Tirado Jiménez, Ramón (1994), “La innovación tecnológica en la industria de la informática y las telecomunicaciones en México", Comercio Exterior, vol. 44, núm. 8, agosto, pp. 716-724, México, BANCOMEXT.

Tirole, Jean (1988), The theory of industrial organization, Cambridge, Mass., The MIT Press.

Varian, Hal R. (1992), Microeconomic analysis, Nueva York, W. W. Norton \& Company, tercera edición.

Warman, José (1994), "La competitividad de la industria electrónica: situación y perspectivas”, en Fernando Clavijo y José Casar, La industria mexicana en el mercado mundial. Elementos para una política industrial, México, FCE, col. Lecturas 80 de El Trimestre, pp. 395-496.

y Margaret Miller (1988), Competitividad de la industria electrónica mexicana: estudios de caso, México, Documentos de Trabajo de la Fundación Friedrich Ebert. 